Während das Sammeln von Daten und Kontrollen aller Art in Mode sind, macht sich das Schweizerische Institut für ärztliche Weiter- und Fortbildung SIWF stark für eine möglichst einfache Kontrolle, wofür ihm jedermann dankbar sein kann.

Die Informatik und das Internet bieten denn auch Möglichkeiten, von denen auch Ärztinnen und Ärzte profitie- ren können, um ihren Papierkram ein wenig zu vereinfachen, wie uns Werner Bauer, Präsident des SIWF, nachstehend erklärt. Ihm gebührt Dank für dieses an wichtigen Informationen reiche Editorial.

Dr. med. Jacques de Haller, Präsident der FMH

\title{
Ärztliche Fortbildung zwischen «Erbsenzählen» und Selbstverantwortung
}

Viele von uns haben der reglementierten Fortbildung und den jährlich zu sammelnden Credits gegenüber gemischte Gefühle. Selbstverständlich ist uns die Verpflichtung bewusst, unser Wissen auf dem aktuellen Stand zu halten. Die meisten von uns sind ohnehin neugierig und interessiert, zu erfahren und zu lernen, welche neuen Erkenntnisse in die praktische Tätigkeit einbezogen werden müssen und welche bestandene Evidenz immer noch gilt. Wir wissen natürlich auch, dass Gesetze, Reglemente und eben auch Fortbildungsprogramme erlassen werden, um sicherzustellen, dass auch diejenigen die Anforderungen erfüllen, die es aus eigener Überzeugung nicht täten. Ganz besonders gilt dies für alle Aspekte der Qualitätsförderung, zu der die ärztliche Fortbildung nun einmal gehört. Seit 2007 ist sie denn auch formell eine im Medizinalberufegesetz geregelte Berufspflicht. Was folgt daraus in der Logik der Aufsichtsbehörden? Keine Pflicht ohne Kontrolle! Uns bleibt nichts anderes übrig, als uns damit abzufinden, auch wenn wir das Einsammeln und Abzählen von Credits manchmal als unwürdig erleben mögen. Allerdings bin ich überzeugt, dass die allermeisten Ärztinnen und Ärzte das Stundenziel auch ohne Zählrahmen problemlos erreichen. Allfällige Sanktionen liegen übrigens in der Kompetenz der kantonalen Gesundheitsbehörden.

\section{Online-Fortbildungsplattform für Ärzte: mit wenig Aufwand zum Fortbildungs- diplom}

Was kann das Schweizerische Institut für ärztliche Weiterund Fortbildung SIWF zusammen mit den Fachgesellschaften dazu beitragen, dass die «Erbsenzählerei» uns alle möglichst wenig belastet und die Selbstverantwortung Kernelement der Fortbildung bleibt?

Der Artikel «Ärztliche Fortbildung: Eine neue Ära hat begonnen» auf Seite 1981 dieser Ausgabe der Schweizerischen Ärztezeitung erläutert, mit wie wenig administrativem Aufwand die Fortbildungsprogramme umgesetzt werden können.

Das SIWF versteht sich als Dienstleistungs-Institut für die Fachgesellschaften und hat deshalb eine Online-Fortbildungsplattform (www.fmh.ch $\rightarrow$ SIWF $\rightarrow$ Fortbildung $\rightarrow$
Fortbildungsplattform) entwickelt, die es den Ärzten einfach macht, ihre Aktivitäten zu erfassen, ihrer Fachgesellschaft einzureichen und in einem Zug das Fortbildungsdiplom auszudrucken. Mit der SGIM ist die Plattform erfolgreich gestartet.Wir hoffen, dass sich im Laufe des Jahres 2011 alle anderen Fachgesellschaften anschliessen werden; ihr administrativer Aufwand reduziert sich dann auf ein Minimum.

Auch das Sammeln der erforderlichen 50 Credits pro Jahr (bzw. der 150 Credits über die dreijährige Fortbildungsperiode) wird vereinfacht: Die Fachgesellschaften definieren

\section{Administrative Entlastung für Fachgesellschaften}

ihre fachspezifische Kernfortbildung, von der 25 Credits jährlich gefordert werden. Weitere 25 Credits kann man sich bei Veranstaltungen gutschreiben lassen, die im Rahmen der erweiterten Fortbildung von anderen Fachgesellschaften, von kantonalen Ärztegesellschaften oder von der FMH anerkannt worden sind. Dazu kommen 30 Stunden Selbststudium, die keiner Kontrolle unterliegen. Kollegen mit mehr als einem Facharzttitel erfüllen die Fortbildungspflicht in dem einen Fachgebiet, das ihrer effektiven Tätigkeit am besten entspricht. Schwerpunkte haben keine eigenen Fortbildungsprogramme; für die meisten Fähigkeitsausweise gelten Rezertifizierungsverpflichtungen, die unabhängig von den Fortbildungsprogrammen für die Facharzttitel geregelt sind.

Mit der aufs Wesentliche konzentrierten Fortbildungsordnung und der Online-Fortbildungsplattform stellt das SIWF den Fachgesellschaften und Ärzten eine hilfreiche und nützliche Dienstleistung für das lebenslange Lernen zur Verfügung - so wie Osler es sich vorgestellt hat:

"If the license to practise meant the completion of his education how sad it would be for the practitioner, how distressing for his patients! More clearly than any other the physician should illustrate the truth of Plato's saying that education is a life-long process.» (William Osler, Lancet, 1900).

Dr. med. Werner Bauer, Präsident des Schweizerischen Instituts für ärztliche Weiter- und Fortbildung SIWF 